\title{
PREDICTIVE PARAMETERS OF VENTRICULAR ARRHYTHMIAS IN ACUTE MYOCARDITIS WITH PRESERVED LVEF
}

\author{
Giuseppina Novo ${ }^{1}$, DANIELA DI LISI ${ }^{2}$, Diego Bellavia ${ }^{3}$, Eluisa La Franca ${ }^{3}$, Maria \\ Gabriella Carmina ${ }^{4}$, Salvatore Novo ${ }^{5}$, Gianluca Di Bella ${ }^{6}$, and Francesco Clemenza ${ }^{3}$ \\ ${ }^{1}$ Affiliation not available \\ ${ }^{2}$ Cardiology, A.O.U. Policlinico P. Giaccone, Palermo, Italy \\ ${ }^{3}$ ISMETT \\ ${ }^{4}$ Villa Sofia Cervello United Hospitals \\ ${ }^{5}$ Policlinico di Palermo \\ ${ }^{6}$ Università degli Studi di Messina
}

August 7, 2021

\begin{abstract}
Background: Myocarditis have variable clinical presentation, evolution and prognosis. Aim of our study was to evaluate the value of speckle tracking echocardiography and cardiac magnetic resonance (CMR) in the prediction of ventricular arrhythmias and other cardiovascular adverse events in patients with acute myocarditis, at hospital admission. Methods: 70 patients (62 M, 8 F; mean age 31,3 $\pm 13,2$ ) with myocarditis and preserved left ventricle ejection fraction (LVEF) were enrolled. Electrocardiogram (ECG), continuous ECG monitoring, echocardiography with measurement of global longitudinal strain of the left ventricle (GLS), mechanical dispersion (MD) and CMR with quantitative measurement of delayed enhancement (DE) were performed. Adverse events were assessed (arrhythmias, heart failure, cardiogenic shock, syncope) during in-hospital stay. Results: We found a significant greater amount of DE mass in patients with cardiac arrhythmias $(p=0,01)$, but not of edema $(p=0,57)$. GLS was significantly impaired in patients with ventricular arrhythmias $(\mathrm{p}=0,04)$, conversely MD was not significantly prolonged in this setting $(p=0,16)$. GLS $>-19.2 \%$ (sens $100 \%$, specif $55,7 \%$ ) and a DE mass $>9,9$ gr (sens $100 \%$; specif. $58,6 \%$ ) had the best sensitivity and specificity to identify patients with cardiac arrhythmias. Compared to GLS, DE mass showed a stronger association with ventricular arrhythmias $(\mathrm{p}<0,001)$. Conclusions: in our study DE mass and GLS were associated with ventricular arrhythmias in patients with acute myocarditis and preserved LVEF. DE showed the stronger association with the occurrence of ventricular arrhythmias.
\end{abstract}

\section{Hosted file}

manuscript myocarditis 07:21.docx available at https://authorea.com/users/429572/articles/ 533260-predictive-parameters-of-ventricular-arrhythmias-in-acute-myocarditis-withpreserved-lvef 

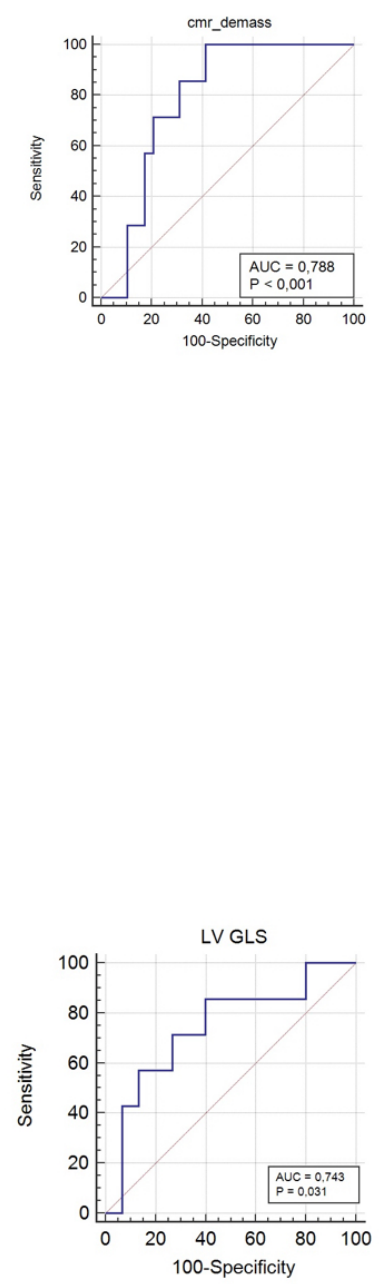